to address both parent and infant needs. Careful preparation for discharge and good follow-up arrangement after discharge may reduce these risks. Comprehensive discharge planning includes assessment of the neonate's readiness for discharge and preparedness of families to care of their infant at home. Objectives Applying the concept of Family centered care increasingly offers families opportunity to participate in caregiving and decision making throughout their hospital stay, gradually building their confidence and competence.

We aimed to improve the family participation in discharge process and reduce the percentage of cases being discharged post 4 hours from decision of discharge from 49\%(our base line preintervention) to less than $20 \%$ in $12-18$ months and reduce the readmission(in $<72$ hrs) rates to zero.

Methods A task force was formed to develop a comprehensive discharge readiness checklist. Discharge checklist contained various components to tick and sign by a team member/parent starting from 2 weeks prior to discharge till the day of discharge. It was reviewed daily during the ward rounds. As per the checklist parents were educated about medications, basic life support training, warning signs and symptoms of illness etc.

This checklist was continuously audited with PDSA cycles and interventions introduced to correct the problems resulting in delayed/incomplete discharges.

Parents were given a discharge folder that included all teaching handouts, medication sheets, growth charts, supplies, follow-up appointments with confirmed dates, relevant phone contacts and a copy of the discharge summary.

Results We achieved $>95 \%$ compliance with the discharge process checklist and a reduction in delayed discharges from $49 \%$ to $2 \%$. we also achieved zero readmission rates

Conclusions Discharge process requires a multi-disciplinary approach. Development of a comprehensive discharge planning toolkit facilitated the discharge process. This tool kit also enabled us in early identification of chronic cases nearing discharge.

We recommend that NICU should form a discharge facilitation task force and develop a discharge checklist/toolkit according to their available resources. This will not only facilitate timely discharges but also has a positive impact on staff and parental satisfaction, bed occupancy rates, cost savings, and better patient flow. We believe this process makes parents a stakeholder in the care of the baby. We will be happy for any unit to contact us for further information and guidance.

\section{THE ROLE OF FECAL CALPROTECTIN IN PREDICTING ACTIVITY OF INFLAMMATORY BOWEL DISEASE IN PEDIATRIC AGE GROUP IN DUBAI HOSPITAL, UAE: 2012- 2016}

Khalid Abdalbagi, Khalid Abdalbagi. United Arab Emirates

\subsection{6/bmjpo-2021-RCPCH.45}

Background Inflammatory bowel disease (IBD) is affecting patients at increasingly younger ages. Endoscopy (upper/lower) with histopathology remains to be the gold standard for the diagnosis. Biochemical markers, as endoscopy is sort of challenging in children, were more and more evaluated in order to define children with high likelihood of needing such invasive procedure. Fecal calprotectin (FC) is raised in patients with IBD. However, it is also raised in other causes of intestinal inflammation. On top of that Studies evaluating FC during the initial investigation of children with suspected IBD have been limited, especially with regard to their small patient groups.

Objectives We aimed to evaluate the diagnostic accuracy of FCin diagnosing IBD patients in comparison with those who had other (non IBD) GIT stressors. Also to define a level at which FC can be safely relied on to distinguish between both. Methods Using a retrospective case-control design all podiatric patients $(<14$ years) who underwent endoscopy in the period between January, 2012 to May 2016 were reviewed. All IBD and non-IBD patients who had a FC measurement available before or within 6 months of endoscopic evaluation were examined and FC results were obtained. FC was measured using the PhiCal Test. SPSS version 23 for windows were used to statistically analyse the results.

Results A total of 138 patients (45 IBD and 93 non-IBD controls) met the inclusion criteria. The median FC at diagnosis for IBD group was $1360 \mathrm{mg} / \mathrm{L}$ IQR: (556.5 -4085 mg/L), compared to median FC of $91 \mathrm{mg} / \mathrm{L}$ IQR: $(34.5-277.5 \mathrm{mg} / \mathrm{L})$ in the control group (P. value $=0.2653)$. There was no significant difference between different types of IBD (P.value = 0.24).Significant difference was noticed between the 2 groups when FC was taken at levels $\geq 850 \mathrm{mg} / \mathrm{L}$ with sensitivity 66.7\% (95\% CI: 51.1-80) and specify $87.1(95 \%$ CI: 78.693.2)

Conclusions This study validates FC as a highly sensitive marker for gut inflammation in general when low cut-off levels are taken. However it is poorly specific for IBD with significant result being only observed at relatively high cut-off values. Thus, FC can safely rule out IBD yet further investigations are needed when FC are above normal.

\section{REVERSAL OF STUNTING IN HOLISTIC HEALTHCARE- EDUCATION PROGRAMME IN ODISHA STATE, INDIA}

Abigail Nye, Catherine Morris, Mary Cusack. UK

\subsection{6/bmjpo-2021-RCPCH.46}

Background Stunted childhood growth is a global problem, and is a major cause of morbidity and mortality. Its causes are complex and multi-factorial, reflecting chronic undernutrition during crucial periods of growth and development in early life. Its consequences are evident at the individual, family and societal level, and can extend into future generations.

A child has stunting if their height-for-age z-score (HAZ) is below 2 standard deviations (SD) from the median of the World Health Organisation (WHO) Child Growth Standards. Until recently it was thought that children could not recover from stunting after the age of 2 years, but this has been refuted by several studies.

Objectives We aimed to investigate the impact on children's height and weight, following enrolment in a free programme offering holistic healthcare, education and play, in Odisha state, India.

Methods We conducted a retrospective observational study analysing anthropometric data for children who are enrolled in the Love the One (LTO) programme in Odisha, India. LTO provides a holistic approach to education, healthcare and childcare for poor and deprived children. Children's height and weight were collected at admission to the programme, and annually thereafter for 5 years, and converted to a centile 